\title{
Parent Emigration, Physical Health and Related Risk and Preventive Factors of Children Left Behind: A Systematic Review of Literature
}

\author{
Justina Račaitė $^{1, *}$, Jutta Lindert ${ }^{2,3}$, Khatia Antia ${ }^{4}$, , Volker Winkler ${ }^{4}$, Rita Sketerskiené ${ }^{1}$, \\ Marija Jakubauskienè ${ }^{1}$, Linda Wulkau ${ }^{2,5}$ and Genè Šurkienè ${ }^{1}$
}

\section{check for}

updates

Citation: Račaitè, J.; Lindert, J.; Antia, K.; Winkler, V.; Sketerskienè, R.; Jakubauskienė, M.; Wulkau, L.; Šurkienè, G. Parent Emigration, Physical Health and Related Risk and Preventive Factors of Children Left Behind: A Systematic Review of Literature. Int. J. Environ. Res. Public Health 2021, 18, 1167. https:// doi.org/10.3390/ijerph18031167

Academic Editors: Ko-Ling Chan and David Schwebel

Received: 4 January 2021

Accepted: 22 January 2021

Published: 28 January 2021

Publisher's Note: MDPI stays neutral with regard to jurisdictional claims in published maps and institutional affiliations.

Copyright: (c) 2021 by the authors. Licensee MDPI, Basel, Switzerland. This article is an open access article distributed under the terms and conditions of the Creative Commons Attribution (CC BY) license (https:/ / creativecommons.org/licenses/by/ $4.0 /)$.
1 Department of Public Health, Institute of Health Sciences, Faculty of Medicine, Vilnius University, M.K. Čiurlionio Str. 21, LT-03101 Vilnius, Lithuania; rita.sketerskiene@mf.vu.lt (R.S.); marija.jakubauskiene@mf.vu.lt (M.J.); gene.surkiene@mf.vu.lt (G.Š.)

2 Department of Social Work and Health, University of Applied Sciences Emden/Leer, Constantiaplatz 4 , 26723 Emden, Germany; jutta.lindert@hs-emden-leer.de (J.L.); linda.wulkau@hs-emden-leer.de (L.W.)

WRSC, Brandeis University, Epstein Building, MS 079, 515 South Street, Waltham, MA 02453, USA

4 Heidelberg Institute of Global Health, Heidelberg University Hospital, Im Neuenheimer Feld 130/3, 69120 Heidelberg, Germany; khatia.antia@uni-heidelberg.de (K.A.); volker.winkler@uni-heidelberg.de (V.W.)

5 Institute for Epidemiology, Social Medicine and Health Systems Research, Hannover Medical School, Carl-Neuberg-Straße 1, 30625 Hannover, Germany

* Correspondence: justina.racaite@mf.vu.lt; Tel.: +370-63158873

\begin{abstract}
The aim of our study was to systematically review the literature on physical health and related consequences of internal and international parental migration on left-behind children (LBC). This review followed PRISMA guidelines. We searched the PubMed, Web of Science, Academic Search Complete, PsycINFO, and Cochrane databases and included studies reporting physical health-related outcomes of children affected by parental migration. The quality of the studies was assessed using the Quality Assessment Tool for Observational Cohort and Cross-Sectional Studies. We selected 34 publications from a total of 6061 search results. The study found that LBC suffer from poor physical health as compared with non-LBC. Physical health-related risk factors such as underweight, lower weight, stunted growth, unhealthy food preferences, lower physical activity, smoking, alcohol consumption, injuries, and incomplete vaccination tend to be more prevalent among LBC in China. Studies focussing on international migration argue that having migrant parents might be preventive for undernutrition. Overall, our study showed that children affected by internal or international migration tend to have similar physical health outcomes. Moreover, we identified a lack of evidence on international parental migration that may have influenced the overall impacts. Further studies addressing international migration would contribute to better understand the impacts of migration for LBC.
\end{abstract}

Keywords: children left behind; parental migration; physical health; children health

\section{Introduction}

The United Nation's Convention on the Rights of the Child " [recognized] that the child, for the full and harmonious development of his or her personality, should grow up in a family environment, in an atmosphere of happiness, love and understanding" [1]. The attachment theory, formulated by John Bowlby, states that for the successful social and emotional development, every child needs a close relationship with at least one primary caregiver [2]. However, there are multiple reasons for parental absence, such as divorce or death. Moreover, children are sometimes taken from unsafe family environments temporarily or permanently.

The migration of parents is another form of child separation from one or both parents. The International Organization for Migration defines migration as "the movement of a 
person or a group of persons, either across an international border, or within a State [independent of] its length, composition and causes [including] migration of refugees, displaced persons, economic migrants, and persons moving for other purposes" [3]. The World Migration Report has reported that, in 2019, the number of international migrants globally was 272 million, which is around 3.5\% of the world's population [4]. Migration is important for the economic growth and improvement of countries. The overwhelming majority of people choose to migrate internationally for reasons related to work, family, and study. However, events such as a conflict, persecution, and disaster force people to migrate without having any choice [4]. Safe, orderly, and regular international migration is included in the 2030 Agenda for Sustainable Development Goals [5].

Employment migration may cause a number of short- and long-term consequences. One of them is the separation of families where children are left behind in their region or countries of origin. In the available literature, children left behind are defined as individuals below the age of 18 , whose parent(s) migrate to other places for work for at least six months [6,7]. Thousands of children are considered to be left behind in many lowand middle-income countries. For example, it has been estimated that in the Philippines, $27 \%$, Ecuador, $36 \%$, and rural South Africa, $40 \%$ of children have at least one migrant parent [8].

Health issues of left-behind children are increasingly being discussed in the scientific literature. Some authors have conducted systematic literature reviews focussing mostly on rural-urban migration in China [8-11]. Previous studies in this field have made an important contribution regarding the understanding of how parental migration affects the social environment and psychological well-being of LBC, education, and health $[8,12-14]$. Following scientific interest, the United Nations Children's Fund (UNICEF) recently drew attention to the vulnerability of these children [15]. This issue has been well explored in China, where migration happens internally, from rural to urban areas. Despite the increased attention, there is a lack of data on the impact of international parental migration on LBC's physical health outcomes. Our study seeks to analyse and synthesize the most recent evidence on health consequences of internal and international parental migration on LBC's physical health and related risk and preventive factors.

\section{Materials and Methods}

For this study, we followed the Preferred Reporting Items for Systematic Reviews and Meta-Analyses (PRISMA) guidelines [16].

We searched PubMed, PsycINFO, Web of Science, Academic search complete, and Cochrane databases for relevant studies published up to 15 May 2020. Our search was guided by the concept of population, exposure, comparator, and outcomes (PECO). We applied the following search terms: left alone OR left behind and stay at home OR left over AND child* AND parent* AND emigrant* OR migrant household AND physical* health OR overweight OR obesity OR stunting OR vaccination OR breastfeed* OR "physical* activity". In addition, we searched the reference lists of included studies and relevant systematic reviews.

We included studies based on the following criteria: (1) study population children (below age 18), (2) original study, (3) one or both parents live in internal or international migration, (4) quantitative measure of physical health outcomes on children, (5) available in English. Two authors (J.R. and R.S.) independently performed title and abstract screening. Disagreements were solved by discussions with a third opinion (G.S.). Studies with the following criteria were excluded: published before 1 January 2008; qualitative or experimental studies; mental health, well-being or educational outcomes; and children living in migration together with parents.

Two authors (J.R. and R.S.) extracted the following information from included papers: first author; year of publication; geographical area; study design; sample size and method, age and gender distribution, definitions and measures of exposures and outcomes, results, covariates and limitations. Disagreement was solved by including a third opinion (G.Š.). 
In this systematic review, we analysed studies from the following two perspectives: (1) type of migration (internal/international) outcomes and (2) physical health outcomes and related risk and preventive factors (weight and height, nutrition, health behaviour, injuries, immunization).

The quality of the studies was assessed using the Quality Assessment Tool for Observational Cohort and Cross-Sectional Studies (National Heart, Lung, and Blood Institute, 2014). Assessment consisted of 14 questions. Studies were defined as "good", "fair", or "poor" according to the number of "yes" answers in the evaluation from $50 \%, 49-21 \%$, and below $20 \%$, respectively. Two reviewers (J.R. and L.W.) independently rated the studies and disagreements were resolved by consensus including a third opinion (G.Š.).

\section{Results}

\subsection{Study Selection and Characteristics}

We identified 6061 studies by searching the databases, from which 1386 were duplicates. We excluded 4597 records after title and abstract screening. Six articles were added after searching the reference lists of included studies. Full-text reading of 84 articles led to the exclusion of 50 manuscripts. We included 34 studies published between 1 May 2008 and 12 March 2020 in the final analysis. Reasons of exclusion are given in Figure 1.

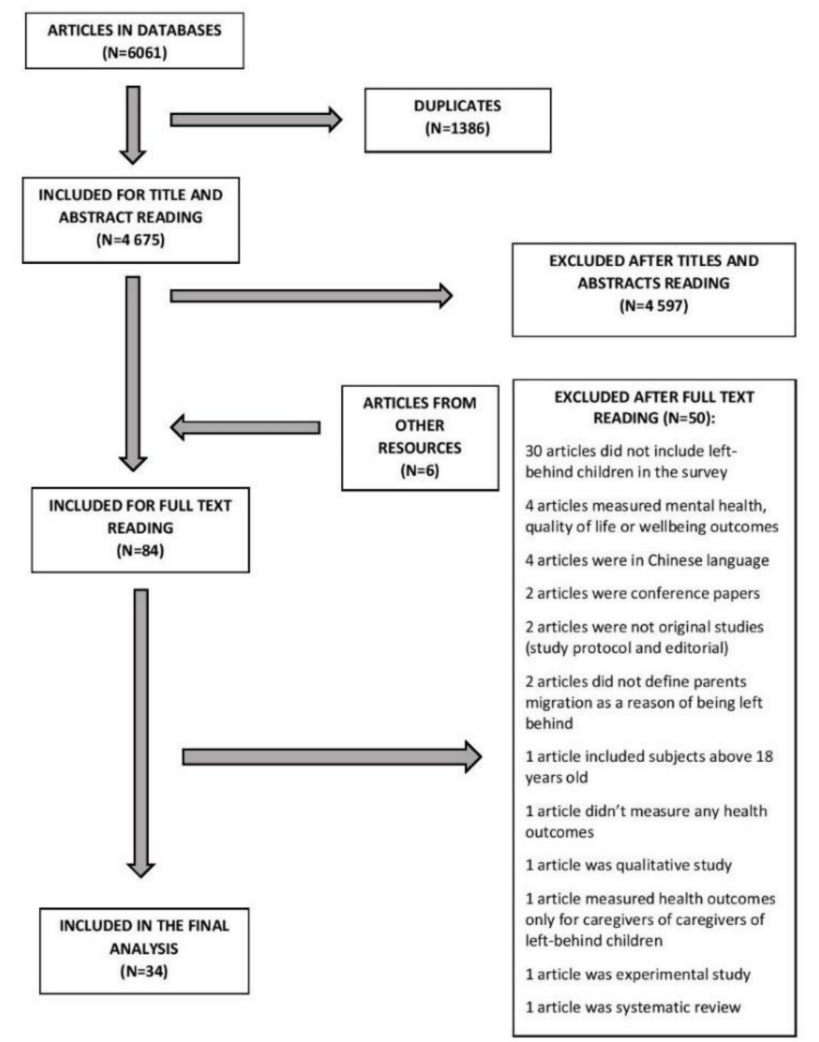

Figure 1. Study selection scheme.

Included studies were cross-sectional (26) or longitudinal (8). The majority of studies (25) were conducted in China. Studies conducted in other countries included the following: two studies from Mexico [17,18]; two studies from Sri Lanka [19,20]; one study from the Philippines [21]; one study from Bangladesh [22]; one study from the Philippines and Vietnam [23]; one study from Ethiopia, India, Peru, and Vietnam [24]; one study from Moldova and Georgia [25]. We evaluated 30 studies as "good" and four studies as "fair". The detailed characteristics of included studies are provided in Table 1. Table 2 describes the main outcome measures. 
Table 1. Characteristics of the studies.

\begin{tabular}{|c|c|c|c|c|c|c|c|c|}
\hline No & $\begin{array}{l}\text { First Author, Year } \\
\text { of Publication }\end{array}$ & Country & Study Design & Sample Size (N) & $\begin{array}{c}\text { Age (Range, Mean, } \\
\text { SD) }\end{array}$ & $\begin{array}{c}\text { Gender Distribution (Male; } \\
\%)\end{array}$ & Outcomes & Quality Rating ${ }^{1}$ \\
\hline 1. & Ban, 2017 [26] & China & Cross-sectional & 6136 & 0-35 months & $\begin{array}{c}\text { Neither parent migrated } \\
54.3 \% \text {, father migrated only } \\
52.7 \% \text {, mother with/without } \\
\text { father migrated } 54.2 \%\end{array}$ & $\begin{array}{l}\text { 1. Stunting } \\
\text { 2. Breastfeeding } \\
\text { 3. Milk feeding }\end{array}$ & Good \\
\hline 2. & Cebotari, 2018 [25] & Moldova, Georgia & Cross-sectional & $\begin{array}{l}\text { Moldova (1601), } \\
\text { Georgia (1193) }\end{array}$ & $\begin{array}{c}\text { 10-18 years Moldova } \\
14.3 \text { (SD 2.59), Georgia } \\
13.44 \text { (SD 2.40) }\end{array}$ & $\begin{array}{c}\text { Moldova } 51.93 \% \text {, Georgia } \\
53.96 \%\end{array}$ & Child health status & Good \\
\hline 3. & $\begin{array}{l}\text { Edelblute, } 2019 \\
{[17]}\end{array}$ & Mexico & Cross-sectional & 542 & 5.3 (SD 2.96) & $\begin{array}{c}\text { Father present 51.5\%, father } \\
\text { absence (migration) } 48.1 \%, \\
\text { father absence (other reasons) } \\
42.9 \%\end{array}$ & $\begin{array}{l}\text { Maternal ratings } \\
\text { of child poor } \\
\text { health }\end{array}$ & Good \\
\hline 4. & Gao, 2013 [27] & China & Cross-sectional & 2558 & 13.8 (SD 1.14) & $55 \%$ & $\begin{array}{l}\text { 1. Past } 30 \text { days } \\
\text { smoking } \\
\text { 2. Self-efficacy of } \\
\text { smoking }\end{array}$ & Good \\
\hline 5. & Gao, 2010 [6] & China & Cross-sectional & 2986 & $\begin{array}{c}10-18 \text { years } 14.2(\mathrm{SD} \\
1.4)\end{array}$ & $51.4 \%$ & $\begin{array}{l}\text { 1. Health-related } \\
\text { behaviours } \\
\text { 2. Nutritional } \\
\text { status }\end{array}$ & Good \\
\hline 6. & Graham, 2013 [23] & $\begin{array}{l}\text { The Philippines, } \\
\text { Vietnam }\end{array}$ & Cross-sectional & $\begin{array}{l}\text { The Philippines } \\
\text { (480), Vietnam } \\
\text { (482) }\end{array}$ & 9-11 years & $\mathrm{NA}^{2}$ & Stunting & Good \\
\hline 7 & Guo, 2017 [28] & China & Longitudinal & 6083 & 12.27 (SD 3.71) & $55 \%$ & \multirow{2}{*}{$\begin{array}{l}\text { Self-rated health } \\
\text { Haemoglobin } \\
\text { concentration }\end{array}$} & Good \\
\hline 8. & $\begin{array}{c}\text { Hipgrave, } 2014 \\
\text { [29] }\end{array}$ & China & Cross-sectional & 2244 & 6-23 months & $56.3 \%$ & & Good \\
\hline 9. & $\mathrm{Hu}, 2018$ [30] & China & Cross-sectional & 4479 & $6-16$ years & $46.5 \%$ & $\begin{array}{l}\text { Unintentional } \\
\text { injuries }\end{array}$ & Good \\
\hline 10. & Huang, 2018 [7] & China & Cross-sectional & 916 & 11.6 & $57 \%$ & $\begin{array}{l}\text { Children's health } \\
\text { conditions }\end{array}$ & Good \\
\hline 11. & Islam, 2019 [22] & Bangladesh & Cross-sectional & 23,402 & $0-5$ years 2 & $51.3 \%$ & $\begin{array}{l}\text { 1. Stunting } \\
\text { 2. Wasting } \\
\text { 3. Underweight } \\
\text { 4. Nutritional } \\
\text { disorders }\end{array}$ & Good \\
\hline
\end{tabular}


Table 1. Cont.

\begin{tabular}{|c|c|c|c|c|c|c|c|c|}
\hline No & $\begin{array}{l}\text { First Author, Year } \\
\text { of Publication }\end{array}$ & Country & Study Design & Sample Size (N) & $\begin{array}{l}\text { Age (Range, Mean, } \\
\text { SD) }\end{array}$ & $\begin{array}{c}\text { Gender Distribution (Male; } \\
\% \text { ) }\end{array}$ & Outcomes & Quality Rating ${ }^{1}$ \\
\hline 12. & Jayatissa, 2016 [19] & Sri Lanka & Cross-sectional & 7500 & $\begin{array}{c}\text { 6-59 months } 32.9 \text { (SD } \\
14.7)\end{array}$ & $50.2 \%$ & $\begin{array}{l}\text { 1. Stunting, } \\
\text { wasting and } \\
\text { underweight } \\
\text { 2. Health status } \\
\text { 3. Food intake }\end{array}$ & Fair \\
\hline 13. & Jiang, 2015 [31] & China & Cross-sectional & 1367 & $12.2(\mathrm{SD} 1.3)$ & $56 \%$ & $\begin{array}{l}\text { Current alcohol } \\
\text { drinking }\end{array}$ & Good \\
\hline 14. & Lei, 2018 [32] & China & Cross-sectional & 5413 & $1-15$ years & $\begin{array}{l}\text { Rural without migrant } \\
\text { parents } 55 \% \text {;left behind } 56 \% \text {. }\end{array}$ & $\begin{array}{l}\text { 1. Child health } \\
\text { status } \\
\text { 2. Child health } \\
\text { status assessed by } \\
\text { guardians }\end{array}$ & Good \\
\hline 15. & Li, 2015 [33] & China & Longitudinal & 13,171 & 9755 (SD 4.9) & $53 \%$ & $\begin{array}{l}\text { 1.Sickness } \\
\text { 2. Injuries } \\
\text { 3. Chronic } \\
\text { conditions } \\
\text { 4. Acute disease }\end{array}$ & Good \\
\hline 16. & Luo, 2008 [34] & China & Cross-sectional & 1548 & $\begin{array}{l}\text { 0.33-6.92 years } 3.51 \\
\text { (SD 1.59) }\end{array}$ & $56.2 \%$ & $\begin{array}{l}\text { 1. Anthropometry } \\
\text { 2. Dietary intake } \\
\text { 3. Haemoglobin } \\
\text { concentration }\end{array}$ & Fair \\
\hline 17. & Mo, 2016 [35] & China & Cross-sectional & 735 & $\begin{array}{c}1-6 \text { years } \\
4.58 \text { years (SD } 55.0 \\
\text { months) }\end{array}$ & $44.1 \%$ & Physical health & Good \\
\hline 18. & Nguyen, 2016 [24] & $\begin{array}{l}\text { Ethiopia, India, } \\
\text { Peru, Vietnam }\end{array}$ & Cross-sectional & 7725 & $5-8$ years & $\mathrm{NA}^{2}$ & Health status & Good \\
\hline 19. & Ni, 2017 [36] & China & Cross-sectional & 1368 & $12-72$ months & $85 \%$ & $\begin{array}{c}1 . \text { The full } \\
\text { vaccination rate } \\
2 . \text { The } \\
\text { age-appropriate } \\
\text { vaccinationrate }\end{array}$ & Good \\
\hline 20. & $\begin{array}{c}\text { Palos-Lucio, } 2015 \\
{[18]}\end{array}$ & Mexico & Cross-sectional & 239 & 9-12 years & $\begin{array}{l}\text { Migrant household } 51.69 \% \text {; } \\
\text { Non-migrant household } \\
55.46 \% \text {. }\end{array}$ & 1. Physical activity & Good \\
\hline 21. & Shen, 2009 [37] & China & Cross-sectional & 3019 & 5-17 years & $60.3 \%$ & $\begin{array}{l}\text { Unintentional } \\
\text { injuries }\end{array}$ & Fair \\
\hline
\end{tabular}


Table 1. Cont.

\begin{tabular}{|c|c|c|c|c|c|c|c|c|}
\hline No & $\begin{array}{l}\text { First Author, Year } \\
\text { of Publication }\end{array}$ & Country & Study Design & Sample Size (N) & $\begin{array}{c}\text { Age (Range, Mean, } \\
\text { SD) }\end{array}$ & $\begin{array}{c}\text { Gender Distribution (Male; } \\
\% \text { ) }\end{array}$ & Outcomes & Quality Rating ${ }^{1}$ \\
\hline 22. & $\begin{array}{c}\text { Smeekens, } 2012 \\
{[21]}\end{array}$ & The Philippines & Cross-sectional & 205 & $\begin{array}{c}13-18 \\
14.58 \text { (SD 1.04) }\end{array}$ & $31.7 \%$ & Physical health & Good \\
\hline 23. & Tang, 2019 [38] & China & Cross-sectional & 1662 & $12-15$ years & $50.2 \%$ & $\begin{array}{l}\text { 1. Physical health } \\
\text { status } \\
\text { 2. Health } \\
\text { behaviours } \\
\text { 3. Frequency of } \\
\text { not going to } \\
\text { school due to } \\
\text { sickness } \\
\text { 4. Vaccination }\end{array}$ & Good \\
\hline 24. & Tang, 2016 [39] & China & Cross-sectional & 1216 & 18-24 months & $50.2 \%$ & Vaccination status & Good \\
\hline 25. & Tao, 2016 [40] & China & Cross-sectional & 827 & $7-15$ years & $51.2 \%$ & $\begin{array}{l}\text { 1. Nutritive status } \\
\text { 2. Food preference }\end{array}$ & Good \\
\hline 26. & Tian, 2017 [41] & China & Longitudinal & 446 & 11-18 years & $60 \%$ & $\begin{array}{l}\text { 1. Growth } \\
\text { 2. Nutrition }\end{array}$ & Good \\
\hline 27. & Tong, 2015 [42] & China & Longitudinal & 8662 & 10.18 (SD 4.77) & $83.8 \%$ & Childhood illness & Good \\
\hline 28. & Wen, 2016 [43] & China & Longitudinal & 2170 & $7-17$ years & At baseline $53.9 \%$ & Blood pressure & Good \\
\hline 30. & Yang, 2016 [44] & China & Cross-sectional & 1343 & $10-14$ years & $56 \%$ & Smoking & Good \\
\hline 31. & Yue, 2020 [45] & China & Longitudinal & 1802 & 6-30 months & $53.1 \%$ & $\begin{array}{l}\text { 1. Nutritional } \\
\text { status } \\
\text { 2. Anaemia status } \\
\text { 3. General health } \\
\text { 4. Feeding } \\
\text { practises }\end{array}$ & Good \\
\hline 32. & Zhang, 2015 [46] & China & Longitudinal & 2555 & $0-17$ years & $57.3 \%$ & Child growth & Good \\
\hline 33. & $\begin{array}{c}\text { Zhang (a), } 2015 \\
\text { [47] }\end{array}$ & China & Longitudinal & 975 & $1-17$ years & $56.9 \%$ & $\begin{array}{l}\text { 1. Dietary } \\
\text { 2. Macronutrient } \\
\text { intakes }\end{array}$ & Good \\
\hline 34. & Zhou, 2015 [48] & China & Cross-sectional & 141,000 & 3-17 years & $\mathrm{NA}^{2}$ & $\begin{array}{l}\text { 1. Health } \\
\text { 2. Nutrition }\end{array}$ & Fair \\
\hline
\end{tabular}

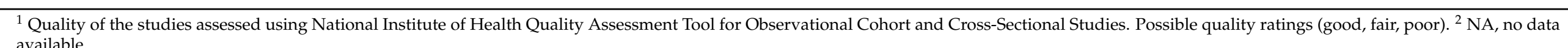
available. 
Table 2. Main outcomes (only statistically significant outcomes provided).

\begin{tabular}{|c|c|c|c|c|c|}
\hline Group & $\begin{array}{l}\text { Outcome (Left-Behind } \\
\text { Children) }\end{array}$ & Sample Size (N) & Covariates $^{2}$ & $\begin{array}{l}\text { Statistics (OR, Mean, SD, } p, 95 \% \text { CI } \\
\text { or Other Statistics If Provided) }{ }^{1}\end{array}$ & Reference \\
\hline \multicolumn{6}{|c|}{ Internal Migration } \\
\hline \multirow{3}{*}{ Physical Health } & More susceptible to illness & 735 & Parenting styles, age of child, health literacy & OR $1.628, p<0.05$ & Mo, 2015 [35] \\
\hline & Childhood illness & 8662 & $\begin{array}{l}\text { Age, gender, household size, income per } \\
\text { capita, grandparents living together, village } \\
\text { context, village size }\end{array}$ & OR 1.29, $\mathrm{SE}=0.164, p<0.05$ & Tong, 2015 [42] \\
\hline & $\begin{array}{l}\text { Pre-hypertension or } \\
\text { hypertension }\end{array}$ & 2170 & $\begin{array}{l}\text { Age, gender, mothers education, fathers } \\
\text { education, annual household per capita } \\
\text { income }\end{array}$ & OR $7.77,95 \%$ CI $2.05-29.4, p<0.01$ & Wen, 2016 [43] \\
\hline \multirow{7}{*}{$\begin{array}{c}\text { Nutrition, Weight and } \\
\text { Height }\end{array}$} & Lower Height for age z-score & 5413 & Age, gender & OR $-0.165, p<0.01$ & Lei, 2018 [32] \\
\hline & Lower Weight for age z-score & 5413 & Age, gender & OR $-0.142, p<0.05$ & Lei, 2018 [32] \\
\hline & Malnutrition rate & 827 & NR/NA & $\begin{array}{c}\text { LBC } 14.83 \%, \text { NoN-LBC } 7.04 \%, p< \\
0.01\end{array}$ & Tao, 2016 [40] \\
\hline & $\begin{array}{l}\text { Less likely to be ever } \\
\text { breastfed }\end{array}$ & 6136 & $\begin{array}{l}\text { Age, gender, ethnicity, elder siblings, guardian } \\
\text { education attainment, the number of } \\
\text { household electrical appliances, year of survey }\end{array}$ & OR $0.30,95 \%$ CI $0.17-0.52$ & Ban, 2017 [26] \\
\hline & Less likely to be breastfed & 1548 & NR/NA & $\begin{array}{c}\text { LBC }(78.7 \%), \text { Non-LBC }(82.8 \%), p< \\
0.05\end{array}$ & Luo, 2008 [34] \\
\hline & Shorter breastfeeding & 6136 & $\begin{array}{l}\text { Age, gender, ethnicity, elder siblings, guardian } \\
\text { education attainment, the number of } \\
\text { household electrical appliances, year of survey }\end{array}$ & $\beta-3.77,95 \%$ CI $-5.01--2.53$ & Ban, 2017 [26] \\
\hline & $\begin{array}{l}\text { Duration of breastfeeding } \\
\text { (months) }\end{array}$ & 1548 & NR/NA & $\begin{array}{l}\text { LBC } \mathrm{M}=9.48, \mathrm{SD}=3.58 ; \text { Non-LBC M } \\
\quad=10.70, \mathrm{SD}=+3.26 ; p<0.001\end{array}$ & Luo, 2008 [34] \\
\hline \multirow[t]{2}{*}{ Unhealthy behaviors } & Alcohol use & 1367 & $\begin{array}{l}\text { Gender, age, grade, if only child in the family, } \\
\text { perceived school performance }\end{array}$ & OR $2.01,95 \%$ CI 1.28-3.16, $p<0.05$ & Jiang, 2015 [31] \\
\hline & Higher smoking rate & 1343 & $\begin{array}{l}\text { Gender, grade, if only child in the family, } \\
\text { perceived school performance }\end{array}$ & OR $5.59,95 \%$ CI $2.38-13.15, p<0.001$ & Yang, 2016 [44] \\
\hline Injuries & $\begin{array}{l}\text { More likely to experience } \\
\text { injury }\end{array}$ & 4479 & $\begin{array}{l}\text { Gender, age, fair physical health, school } \\
\text { academic achievement, only child in the } \\
\text { family, household income level, parental } \\
\text { marital status; maternal education attainment, } \\
\text { family conflicts; model school; peer rejection, } \\
\text { rural region }\end{array}$ & OR 1.208, SE $0.104, p<0.05$ & $\mathrm{Hu}, 2018$ [30] \\
\hline
\end{tabular}


Table 2. Cont.

\begin{tabular}{|c|c|c|c|c|c|}
\hline Group & $\begin{array}{l}\text { Outcome (Left-Behind } \\
\text { Children) }\end{array}$ & Sample Size (N) & Covariates $^{2}$ & $\begin{array}{c}\text { Statistics (OR, Mean, } \mathrm{SD}, p, 95 \% \mathrm{CI} \\
\text { or Other Statistics If Provided) }\end{array}$ & Reference \\
\hline & Higher injury rate & 3019 & NR/NA & $\begin{array}{c}\text { LBC } 252.9 / 1000,95 \% \text { CI 233.0-273.0 } \\
\text { Non-LBC 119.7/1000, 95\% CI } \\
104.9-134.7, p<0.0001\end{array}$ & Shen, 2009 [37] \\
\hline \multirow{3}{*}{ Immunization } & $\begin{array}{l}\text { Lower rates of timely } \\
\text { vaccination }\end{array}$ & 1216 & NR/NA & $\begin{array}{c}\text { LBC55.7\%, 95\% CI 51.3-60.0, } \\
\text { Non-LBC 60.8, 95\% CI 57.3-64.0, } p= \\
0.011\end{array}$ & Tang, 2016 [39] \\
\hline & $\begin{array}{l}\text { Less likely to receive } \\
\text { complete vaccination }\end{array}$ & 1368 & NR/NA & $\begin{array}{c}\text { LBC } 92.7 \%, \text { Non-LBC } 79.9 \%, X^{2}= \\
35.2, p<0.001\end{array}$ & $\mathrm{Ni}, 2017$ [36] \\
\hline & $\begin{array}{c}\text { Lower coverage of complete } \\
\text { vaccination }\end{array}$ & 1662 & NR/NA & $\begin{array}{c}\text { LBC } 38.7 \% \text {, Non-LBC } 44.2 \%, p< \\
0.036\end{array}$ & Tang, 2019 [38] \\
\hline \multicolumn{6}{|c|}{ International Migration } \\
\hline \multirow[b]{2}{*}{ Physical Health } & $\begin{array}{l}\text { Maternal reported child poor } \\
\text { health }\end{array}$ & 542 & Data from model with no covariates added & OR $0.33,95 \%$ CI $0.16-0.7, p<0.01$ & $\begin{array}{c}\text { Edelblute, } 2018 \\
\text { [17] }\end{array}$ \\
\hline & Poorer physical health & 205 & NR/NA & $\begin{array}{c}\mathrm{LBC} \mathrm{M}=5.09, \mathrm{SD}=0.78 ; \mathrm{NoN} \mathrm{LBC} \\
\begin{aligned} \mathrm{M}=5.43, \mathrm{SD}= & 0.63 ; \mathrm{F}(1.199)=9.08 ; p \\
& <0.01\end{aligned}\end{array}$ & $\begin{array}{l}\text { Smeekens, } 2012 \\
{[21]}\end{array}$ \\
\hline Unhealthy behaviors & Lower physical activity & 239 & $\begin{array}{l}\text { Age, gender, body mass index, maternal } \\
\text { schooling, paternal schooling, household } \\
\text { characteristics }\end{array}$ & $\mathrm{OR}-0.56, p<0.05$ & $\begin{array}{l}\text { Palos-Lucio, } \\
2015[18]\end{array}$ \\
\hline
\end{tabular}

${ }^{1}$ LBC, left-behind children; Non-LBC, not left-behind children; ${ }^{2}$ NR/NA, not reported or not applicable. 


\subsection{Internal Migration and Physical Health Outcomes}

In China, five studies found that parental migration had negative consequences on child health $[32,33,35,42,43]$. As compared with children of non-migrant parents, LBC were more susceptible to illness and had a higher prevalence of acute and chronic diseases $[33,35]$. Being a child left behind was also strongly and positively associated with the pre-hypertension or hypertension ( $\mathrm{OR}=7.77, p<0.01)$ [43].

Studies included in our analysis reported contradictory results with respect to physical health outcomes of LBC with one or both migrant parents. In China, findings suggest that a mother's absence alone would not affect a child's health, but both a mother's and father's absence together would have a significant negative effect on LBC in rural China [33]. Children raised by a single parent tend to be more susceptible to illness than children raised by both parents [35]. Among LBC, those living with their mother were more likely to be in better health, than those living with their father only [33].

Regarding the age and a gender of $\mathrm{LBC}$, some studies suggested that left-behind adolescents (13-18 years) could have worse outcomes than younger children [7]. Girls may be more vulnerable than boys to the absence of parental care [33].

One study did not find any significant relationship between parent emigration and child health status [28] and one study found no evidence that children living in migration together with their parents had better health than children left behind [7].

\subsection{Internal Migration and Risk and Preventive Factors}

\subsubsection{Nutrition, Weight, and Height}

Studies included in this review found that, in China, parental migration negatively affected child nutrition [45]. Firstly, as compared with the control group (children of non-migrant parents), children left behind were less likely to receive age-appropriate breastfeeding and the duration of breastfeeding was significantly shorter [26,34]. Total food intake, as well as intake of meat, fish and eggs were lower among LBC [34]. Moreover, children with both parents absent were most likely to skip breakfast, as well as eat high-fat food and sweetened snacks [6,46]. Higher fat and lower protein diet were more common among left-behind boys [46]. Accordingly, more LBC disliked vegetables $(\mathrm{M}=3.66, \mathrm{SD}=0.55)$ and fruits $(\mathrm{M}=3.81, \mathrm{SD}=0.47, p<0.01)$ than non-LBC $(\mathrm{M}=3.89$, $\mathrm{SD}=0.27$ and $\mathrm{M}=3.97, \mathrm{SD} 0.83, p<0.01$ ) [40]. Due to iron-poor food intake, $\mathrm{LBC}$ were at a higher risk of developing anaemia, especially at a younger age [29,34]. Additionally, Zhang et al. found gender differences, for example, on the one hand, LB boys in early childhood showed slower height and weight gain as compared with boys living in non-migrant households [47]. On the other hand, Gao et al. suggested that children left behind might have an increased risk of being overweight [6].

Two studies found no negative impact of parental migration for weight and height outcomes [34,41]. One study found high rates of nutrition problems regardless of parental migration status [48].

\subsubsection{Unhealthy Behaviours}

In general, children with both parents absent were more likely to engage in risky behaviours such as unhealthy diet, physical inactivity, sedentary lifestyle, smoking, and drinking [48].

With respect to addictive behaviours, studies found that alcohol consumption and smoking were higher among children of both migrant parents as opposed to only one (or none) migrant parent $[6,31,44]$. Other authors claimed that maternal migration increased the risk of adolescent smoking, while paternal migration could even protect children from smoking [27]. In terms of gender, some authors found that LB girls were at a higher risk for smoking and binge drinking [6], whereas other studies reported that more LB boys tended to be smokers and current alcohol users than LB girls [31,44]. 


\subsubsection{Injuries}

Some authors have suggested that LBC have a higher risk of getting injured $[30,37]$. The annual injury rate was more than double among LBC as compared with children living with both parents [37]. When controlling for other variables, LBC were more likely to experience unintentional injuries than residential children [30]. The most frequently reported injuries were falls, contact with sharp instrument, striking by objects or person, bitten, or struck by animals, and injuries caused by nature or environment factors [30].

\subsubsection{Immunization}

Studies included in this review found a lower coverage of vaccination among LBC as compared with non-LBC [38]. Children of non-migrating parents (95.7\%) were more likely to receive complete vaccination as opposed to LBC $(79.9 \%, p<0.001)$ [36]. Moreover, LBC had significantly lower coverage of timely vaccination [39].

\subsection{International Migration and Physical Health Outcomes}

Physical health outcomes were analysed in three studies conducted in different regions $[17,21,25]$. A study from the Philippines found that left-behind adolescents ( $M=5.09$, $\mathrm{SD}=0.78)$ reported poorer physical health than non-LBC $(\mathrm{M}=5.43, \mathrm{SD}=0.63), p<0.01)$. The same study found that left-behind adolescents (13-18 years) might be more negatively affected than younger children [21].

A Mexican study compared differences between mother and father emigration and found poorer health outcomes among LBC of international migrant fathers then children of non-migrant fathers [17].

One study conducted in Moldova and Georgia did not find any significant association between parent emigration and child health status [25].

\subsection{International Migration and Risk and Preventive Factors \\ 3.5.1. Nutrition, Weight, and Height}

Regarding weight and height outcomes, findings from Sri Lanka found a lower prevalence of stunting $(11.5 \%$ vs. $14.8 \%)$, wasting $(18.1 \%$ vs. $21.5 \%)$, and underweight ( $24.3 \%$ vs. $26.2 \%$ ) among LBC as compared with non-LBC, respectively [19]. A study from Ethiopia, India, and Peru found higher weight and height and lower proportion of malnourished children in migrant households as opposed to children from non-migrant households [24]. However, another study conducted in the Philippines and Vietnam did not support this [23].

Two studies from Bangladesh and Sri Lanka found no negative impact of parental migration for weight and height outcomes [20,22].

\subsubsection{Physical Activity}

There was only one study from Mexico which found lower physical activity among LBC than non-LBC. Children with parental migration experience had 0.56 less physical active time (hours) per day as compared with children from non-migrant households [18].

\section{Discussion}

In this study, we systematically reviewed the evidence on the effects of internal and international parental migration on their children's physical health outcomes and related risk and preventive factors. By doing so, we provided comparative analysis of the outcomes of the internal rural-urban and international migration. Previous studies in this field analysed the outcomes independently from type of migration [8] or focused on international migration only [14]. Our study was motivated by the substantial research gap in research on international labour migration effects on LBC in many low- and middleincome countries. A predominance of studies focused on internal migration from China have clearly shown the emerging need to shift this paradigm and investigate the issue in a global context [49]. 
As explained above, all studies analysing internal migration outcomes were conducted in China, while all studies from other regions (Americas, South and Southeast Asia, Africa, and the East European Region) examined international parental migration outcomes on LBC. Our study found that, despite the type of migration/region, LBC suffer from poor general health. Children with migrating parents are at a higher risk of developing poor nutrition, overweight or obesity, addictive behaviours, physical inactivity, lower vaccination coverage, and more frequent injuries than non-LBC. Some authors have explained such findings using the cognitive stress theory developed by Lazarus and Folkman, in 1984 [21]. Our findings are consistent with previous studies [8] showing that LBC's physical health outcomes are not improving over time.

Despite the negative outcomes reported, several authors also discuss the potential benefits of parental migration on their children's physical health. Some studies suggested that remittances could prevent undernutrition and improve access to medical care for LBC $[17,19,25]$. In contrast, studies that focused on internal migration from China found that remittances were related to a higher risk of overweight [35].

Studies included in this review found that socioeconomic conditions and characteristics of caregivers play an important role for potential outcomes. The following factors were most reported: parents' and caregivers' education, sex of migrant parent, household size, income per-capita, parental marital status, and siblings. The health literacy of a primary caregiver was found to be essential for nutrition, health, and development of a child [35]. Our study findings show that having a migrant mother might be more harmful, than having a migrant father $[6,19,33]$. Some authors emphasize the negative influence of a culture, for example, traditions of physical punishments in some countries, such as Moldova and Georgia [50]. However, most of the outcomes stay negative and significant after controlling for potential socioeconomic cofounders (Table 2).

In general, studies from China and Mexico found that LBC might be more vulnerable to risky behaviour and an unhealthy lifestyle than non-LBC. LBC affected by internal migration tend to have more risky behaviour such as alcohol consumption, smoking, as well as a high fat and low protein diet [44,47]. A study from Mexico also found lower physical activity among LBC [18]. This shows the need for improving health literacy and health education in schools and among caregivers of LBC.

Several limitations of our study should be mentioned. We included studies published only in English. Most of the included articles focused on Chinese populations, with a few exceptions from the Caribbean region, South America, South Asia, and Europe. Among all the included studies, only nine studies focused on international migration, while all other studies came from China, and therefore addressed only internal migration. Most of the studies were cross-sectional, which did not allow drawing conclusions on causation. Finally, authors noted that according to the International Organization of Migration, labour migration trends have increased significantly in recent years [4]. With this in mind, we decided to included studies published after 1 January 2008 aiming at providing the most recent evidence in this field. However, this could be considered to be a limitation, since we may have missed some relevant studies. Despite these limitations, to the best of our knowledge, this is the first study considering both internal and international migration aspects while examining the effects of parental migration on LBC's physical health.

The importance of migration is growing together with globalization, while millions of children are left behind in their countries of origin. Our findings emphasize the need for preventive actions to address the health of LBC. Various international organizations such as UNICEF have brought attention to this vulnerable groups. Even though scholars have addressed this issue in China, there is an urgent need for more evidence from other labour migration-affected regions of the world. Public health interventions for LBC is needed.

\section{Conclusions}

This study found that both internal and international parental migration is associated with child outcomes such as physical health, nutrition, weight and height, injuries, and 
immunization. In most cases, the consequences for child health are negative, however, in low- and middle-income countries parental migration might also prevent left-behind children from undernutrition. When comparing studies from China and other countries, we found similar outcomes (regardless of internal or international migration). This study highlights the knowledge gap on the topic, especially in Western Asia and the East European Region, and calls for action from governments and international institutions, and the research community to better investigate and address the health needs of children affected by parental migration.

Author Contributions: Conceptualization, J.L., M.J. and J.R.; methodology, G.Š. and J.R.; validation, J.R., R.S. and L.W.; formal analysis, J.R. and R.S.; resources, J.R., K.A. and M.J.; writing—original draft preparation, J.R.; writing-review and editing, J.L., K.A., V.W. and G.Š.; visualization, J.R. and K.A.; supervision, G.Š. All authors have read and agreed to the published version of the manuscript.

Funding: This research received no external funding.

Institutional Review Board Statement: Not applicable.

Informed Consent Statement: Not applicable.

Acknowledgments: Authors wish to thank Vilnius University librarian Julija Lekavičiutè for assistance in article search useful information on databases.

Conflicts of Interest: The authors declare no conflict of interest.

\section{References}

1. United Nations. Convention on the Rights of the Child. 1989. Available online: https://www.ohchr.org/en/professionalinterest/ pages/crc.aspx (accessed on 30 September 2019).

2. Bowlby, J. Attachment: Attachment and Loss Volume One (Basic Books Classics); Basic Books: New York, NY, USA, 1983.

3. International Organization of Migration. Key Migration Terms. 2015. Available online: https://www.iom.int/key-migrationterms (accessed on 30 September 2019).

4. International Organization of Migration. World Migration Report. 2020. Available online: https://publications.iom.int/system/ files/pdf/wmr_2020.pdf (accessed on 8 December 2020).

5. United Nations. The Sustainable Development Agenda. 2016. Available online: https://www.un.org/sustainabledevelopment/ sustainable-development-goals / (accessed on 30 September 2019).

6. Gao, Y.; Li, L.P.; Kim, J.H.; Congdon, N.; Lau, J.; Griffiths, S. The impact of parental migration on health status and health behaviours among left behind adolescent school children in China. BMC Public Health 2010, 10, 56.

7. Huang, Y.; Song, Q.; Tao, R.; Liang, Z. Migration, Family Arrangement, and Children's Health in China. Child. Dev. 2018, 89, e74-e90.

8. Fellmeth, G.; Rose-Clarke, K.; Zhao, C.; Busert, L.K.; Zheng, Y.; Massazza, A.; Sonmez, H.; Eder, B.; Blewitt, A.; Lertgrai, W.; et al. Health impacts of parental migration on left-behind children and adolescents: A systematic review and meta-analysis. Lancet 2018, 392, 2567-2582.

9. Zhao, F.; Yu, G. Parental migration and rural left-behind children's mental health in China: A meta-analysis based on mental health test. J. Child. Fam. Stud. 2016, 25, 3462-3472.

10. Wang, X.; Ling, L.; Su, H.; Cheng, J.; Jin, L.; Sun, Y.H. Self-concept of left-behind children in China: A systematic review of the literature. Child. Care Health Dev. 2015, 41, 346-355.

11. Sun, X.; Chen, M.; Chan, K.L. A meta-analysis of the impacts of internal migration on child health outcomes in China. BMC Public Health 2016, 16, 66.

12. Valtolina, G.G.; Colombo, C. Psychological Well-Being, Family Relations, and Developmental Issues of Children Left Behind. Psychol. Rep. 2012, 111, 905-928.

13. Dillon, M.; Walsh, C.A. Left Behind: The Experiences of Children of the Caribbean Whose Parents Have Migrated. J. Comp. Fam. Stud. 2012, 43, 871.

14. Antia, K.; Boucsein, J.; Deckert, A.; Dambach, P.; Račaitè, J.; Šurkienè, G.; Jaenisch, T.; Horstick, O.; Winkler, V. Effects of International Labour Migration on the Mental Health and Well-Being of Left-Behind Children: A Systematic Literature Review. Int. J. Environ. Res. Public Health 2020, 17, 4335.

15. UNICEF. Working Paper Children "Left-behind". 2019. Available online: https://www.unicef.org/media/61041/file (accessed on 16 January 2020).

16. David Moher, P.; Alessandro Liberati, M.D.; Jennifer Tetzlaff, B.S.; Douglas, G.; Altman, D.S.; the PRISMA Group. Preferred Reporting Items for Systematic Reviews and Meta-Analyses: The PRISMA Statement. Ann. Intern. Med. 2009, $144,364-367$.

17. Edelblute, H.B.; Altman, C.E. Father Absence, Social Networks, and Maternal Ratings of Child Health: Evidence from the 2013 Social Networks and Health Information Survey in Mexico. Matern. Child. Health J. 2018, 22, 626-634. 
18. Palos-Lucio, G.; Flores, M.; Rivera-Pasquel, M.; Salgado-de-Snyder, V.N.; Monterrubio, E.; Henao, S.; Macias, N. Association between migration and physical activity of school-age children left behind in rural Mexico. Int. J. Public Health 2015, 60, 49-58.

19. Jayatissa, R.; Wickramage, K. What Effect Does International Migration Have on the Nutritional Status and Child Care Practices of Children Left Behind? Int. J. Environ. Res. Public Health 2016, 13, 218.

20. Wickramage, K.; Siriwardhana, C.; Vidanapathirana, P.; Weerawarna, S.; Jayasekara, B.; Pannala, G.; Adikari, A.; Jayaweera, K.; Peiris, S.; Siribaddana, S.; et al. Risk of mental health and nutritional problems for left-behind children of international labor migrants. BMC Psychiatry 2015, 15, 39.

21. Smeekens, C.; Stroebe, M.S.; Abakoumkin, G. The impact of migratory separation from parents on the health of adolescents in the Philippines. Soc. Sci. Med. 2012, 75, 2250-2257.

22. Islam, M.M.; Khan, M.N.; Mondal, M.N.I. Does parental migration have any impact on nutritional disorders among left-behind children in Bangladesh? Public Health Nutr. 2018, 22, 95-103.

23. Graham, E.; Jordan, L.P. Does Having a Migrant Parent Reduce the Risk of Undernutrition for Children Who Stay Behind in South-East Asia? Asian Pac. Migr. J. 2013, 22, 315-348.

24. Nguyen, C.V. Does parental migration really benefit left-behind children? Comparative evidence from Ethiopia, India, Peru and Vietnam. Soc. Sci. Med. 2016, 153, 230-239.

25. Cebotari, V.; Siegel, M.; Mazzucato, V. Migration and child health in Moldova and Georgia. Comp. Migr. Stud. $2018,6,3$.

26. Ban, L.; Guo, S.; Scherpbier, R.W.; Wang, X.; Zhou, H.; Tata, L.J. Child feeding and stunting prevalence in left-behind children: A descriptive analysis of data from a central and western Chinese population. Int. J. Public Health 2017, 62, $143-151$.

27. Gao, Y.; Li, L.; Chan, E.Y.; Lau, J.; Griffiths, S.M. Parental migration, self-efficacy and cigarette smoking among rural adolescents in south China. PLOS ONE. 2013, 8, e57569.

28. Guo, Q.; Sun, W.K.; Wang, Y.J. Effect of Parental Migration on Children's Health in Rural China. Rev. Dev. Econ. 2017, 21, 1132-1157.

29. Hipgrave, D.B.; Fu, X.; Zhou, H.; Jin, Y.; Wang, X.; Chang, S.; Scherpbier, R.W.; Wang, Y.; Guo, S. Poor complementary feeding practices and high anaemia prevalence among infants and young children in rural central and western China. Eur. J. Clin. Nutr. 2014, 68, 916-924.

30. Hu, H.; Gao, J.; Jiang, H.; Xing, P. A comparative study of unintentional injuries among schooling left-behind, migrant and residential children in China. Int. J. Equity Health 2018, 17, 47.

31. Jiang, S.; Chu, J.; Li, C.; Medina, A.; Hu, Q.; Liu, J.; Zhou, C. Alcohol consumption is higher among left-behind Chinese children whose parents leave rural areas to work. Acta Paediatr. 2015, 104, 1298-1304.

32. Lei, L.L.; Liu, F.; Hill, E. Labour Migration and Health of Left-Behind Children in China. J. Dev. Stud. 2018, 54, 93-110.

33. Li, Q.; Liu, G.; Zang, W. The health of left-behind children in rural China. China Econ. Rev. 2015, 36, 367-376.

34. Luo, J.; Peng, X.; Zong, R.; Yao, K.; Hu, R.; Du, Q.; Fang, J.; Zhu, M. The status of care and nutrition of 774 left-behind children in rural areas in China. Public Health Rep. 2008, 123, 382-389.

35. Mo, X.; Xu, L.; Luo, H.; Wang, X.; Zhang, F.; Gai Tobe, R. Do different parenting patterns impact the health and physical growth of 'left-behind' preschool-aged children? A cross-sectional study in rural China. Eur. J. Public Health 2016, 26, 18-23.

36. Ni, Z.L.; Tan, X.D.; Shao, H.Y.; Wang, Y. Immunisation status and determinants of left-behind children aged 12-72 months in central China. Epidemiol. Infect. 2017, 145, 1763-1772.

37. Shen, M.; Yang, S.; Han, J.; Shi, J.; Yang, R.; Du, Y.; Stallones, L. Non-fatal injury rates among the "left-behind children" of rural China. Inj. Prev. 2009, 15, 244-247.

38. Tang, D.; Choi, W.I.; Deng, L.; Bian, Y.; Hu, H. Health status of children left behind in rural areas of Sichuan Province of China: A cross-sectional study. BMC Int. Health Hum. Rights 2019, 19, 4.

39. Tang, X.; Geater, A.; McNeil, E.; Zhou, H.; Deng, Q.; Dong, A.; Li, Q. Parental migration and children's timely measles vaccination in rural China: A cross-sectional study. Trop. Med. Int. Health 2016, 21, 886-894.

40. Tao, S.; Yu, L.; Gao, W.; Xue, W. Food preferences, personality and parental rearing styles: Analysis of factors influencing health of left-behind children. Qual. Life Res. 2016, 25, 2921-2929.

41. Tian, X.; Ding, C.; Shen, C.; Wang, H. Does Parental Migration Have Negative Impact on the Growth of Left-Behind Children? New Evidence from Longitudinal Data in Rural China. Int. J. Environ. Res. Public Health 2017, 14, 11.

42. Tong, Y.Y.; Luo, W.X.; Piotrowski, M. The Association Between Parental Migration and Childhood Illness in Rural China. Eur. J. Popul. 2015, 31, 561-586.

43. Wen, M.; Li, K. Parental and Sibling Migration and High Blood Pressure among Rural Children in China. J. Biosoc. Sci. 2016, 48, 129-142.

44. Yang, T.; Li, C.; Zhou, C.; Jiang, S.; Chu, J.; Medina, A.; Rozelle, S. Parental migration and smoking behavior of left-behind children: Evidence from a survey in rural Anhui, China. Int. J. Equity Health 2016, 15, 127.

45. Yue, A.; Bai, Y.; Shi, Y.; Luo, R.; Rozelle, S.; Medina, A.; Sylvia, S. Parental Migration and Early Childhood Development in Rural China. Demography 2020, 57, 403-422.

46. Zhang, N.; Becares, L.; Chandola, T. Does the timing of parental migration matter for child growth? A life course study on left-behind children in rural China. BMC Public Health 2015, 15, 966.

47. Zhang, N.; Becares, L.; Chandola, T. A multilevel analysis of the relationship between parental migration and left-behind children's macronutrient intakes in rural China. Public Health Nutr. 2015, 19, 1913-1927. 
48. Zhou, C.; Sylvia, S.; Zhang, L.; Luo, R.; Yi, H.; Liu, C.; Shi, Y.; Loyalka, P.; Chu, J.; Medina, A.; et al. China's Left-Behind Children: Impact of Parental Migration on Health, Nutrition, And Educational Outcomes. Health Aff. 2015, 34, $1964-1971$.

49. Griffiths, S.M.; Dong, D.; Chung, R.Y. Forgotten need of children left behind by migration. Lancet 2018, 392, 2518-2519.

50. Gassmann, F.; Siegel, M.; Vanore, M.; Waidler, J. Unpacking the Relationship between Parental Migration and Child Well-Being: Evidence from Moldova and Georgia. Child. Indic. Res. 2018, 11, 423-440. 